\title{
Mental health outcomes of ICU and non-ICU healthcare workers during the COVID-19 outbreak: a cross-sectional study
}

\author{
Hannah Wozniak ${ }^{1 *}$ (0), Lamyae Benzakour², Grégory Moullec ${ }^{3}$, Niccolò Buetti ${ }^{4}$, Alice Nguyen ${ }^{3}$, \\ Sandrine Corbaz ${ }^{5,6}$, Pauline Roos ${ }^{6}$, Laure Vieux ${ }^{5}$, Jean-Claude Suard ${ }^{5}$, Rafaël Weissbrodt ${ }^{7}$, Jérôme Pugin ${ }^{1,8}$, \\ Jacques A. Pralong ${ }^{8,9}$ and Sara Cereghetti ${ }^{1+}$
}

\begin{abstract}
Background: Intensive care workers are known for their stressful work environment and for a high prevalence of mental health outcomes. The aim of this study was to evaluate the mental health, well-being and changes in lifestyle among intensive care unit (ICU) healthcare workers (HCW) during the first wave of the COVID-19 pandemic and to compare these results with those of HCW in other hospital units. Another objective was to understand which associated factors aggravate their mental health during the COVID-19 outbreak.

Methods: This cross-sectional survey collected socio-demographic data, lifestyle changes and mental health evaluations as assessed by the Generalized Anxiety Disorder 7 items (GAD-7), the Patient Health Questionnaire 9 items (PHQ9), the Peritraumatic Distress Inventory (PDI) and the World Health Organization Well-Being Index (WHO-5) from the 28th May to 7th July 2020. The study was carried out at Geneva University Hospitals, a group of eight public hospitals in Switzerland. ICU HCW were analyzed for mental health outcomes and lifestyles changes and then compared to non-ICU HCW. A series of linear regression analyses were performed to assess factors associated with mental health scores.

Results: A total of $3461 \mathrm{HCW}$ were included in the study, with 352 ICU HCW. Among ICU HCW, 145 (41\%) showed low well-being, 162 (46\%) symptoms of anxiety, 163 (46\%) symptoms of depression and 76 (22\%) had peritraumatic distress. The mean scores of GAD-7, PHQ-9 and WHO-5 were worse in ICU HCW than in non-ICU HCW $(p<0.01)$. Working in the ICU rather than in other departments resulted in a change of eating habits, sleeping patterns and alcohol consumption $(p<0.01)$. Being a woman, the fear of catching and transmitting COVID-19, anxiety of working with COVID-19 patients, work overload, eating and sleeping disorders as well as increased alcohol consumption were associated with worse mental health outcomes.

Conclusion: This study confirms the suspicion of a high prevalence of anxiety, depression, peritraumatic distress and low well-being during the first COVID-19 wave among HCW, especially among ICU HCW. This allows for the identification of associated risk factors. Long-term psychological follow-up should be considered for HCW.
\end{abstract}

Keywords: Intensive care, Mental health, Caregivers, COVID-19, Healthcare worker, HCW

*Correspondence: hannah.wozniak@hcuge.ch

${ }^{\dagger}$ Jacques A. Pralong and Sara Cereghetti contributed equally to this work

${ }^{1}$ Intensive Care Unit, Geneva University Hospitals, Geneva, Switzerland

Full list of author information is available at the end of the article

\section{Introduction}

Over the last few years, coronaviruses have caused two major pandemics: severe acute respiratory syndrome (SARS) in 2002-2003 and Middle East respiratory syndrome MERS in 2012 [1, 2]. Since March 2020, we are 
facing a new global SARS-CoV-2 pandemic [2] which is having a major impact on society as well as on health care systems around the world.

This unique sanitary situation has forced many hospitals including the Geneva University Hospitals (HUG) in Switzerland to adapt to the increased flow of patients [3]. The HUG, the largest university hospital in Switzerland which comprises eight public hospitals, became a COVID-19 hospital and almost all patients with other pathologies were hospitalized in surrounding private hospitals. The capacity of certain units was increased to admit COVID-19 patients and the intensive care unit (ICU) increased its capacity from 30 to 180 beds. To strengthen the teams, healthcare workers $(\mathrm{HCW})$ from other departments joined the departments most affected by the COVID-19 pandemic, such as intensive care and internal medicine departments. The HUG put in place extraordinary measures to support frontline $\mathrm{HCW}$, such as a psychological support service and hotel beds close to the hospital.

During the SARS epidemic in 2003, Wu et al. showed, in the city of Beijing, China, that $10 \%$ of HCW had posttraumatic stress disorder (PTSD) and that associated risk increased threefold among staff working in close contact with SARS-positive patients [4]. More recently, studies have shown that exposure to a new infectious hazard may generate anxiety, depression or have a negative impact on quality of life [5-7]. Reorganization of work in the context of a crisis can also be a source of stress among employees $[6,8]$. Moreover, psychological consequences for HCW facing a pandemic can be associated to lifestyle changes such as modified dietary and sleeping patterns as well as possible increases in alcohol or tobacco consumption [6].

ICUs are well known for their stressful work environment. Previous studies have shown that ICU nurses are at greater risk of PTSD than nurses working in other units [9] and that ICU HCW have higher levels of anxiety and depression $[8,10]$. Females, nurses, direct exposure to COVID-19 patients and working in the ICU are all factors, which have been found to be associated with negative psychological impact $[6,11,12]$. Additionally, Azoulay et al. identified several factors associated with poorer mental health outcomes during the COVID19 crisis, such as fear of catching the virus, inability to rest, inability to care for loved ones, emotional stress, restricted visitation for relatives of patients and having to witness hasty end-of-life decision-making [8].

In this context, we hypothesize that the COVID-19 pandemic is associated with poor mental health outcomes in HCW and especially those in the ICU. Our primary objective was to assess the mental health, wellbeing and changes in health behaviors of ICU HCW and to make comparisons across different professions (nurses, physicians, care assistants and others), following the first wave of the pandemic. Our secondary objectives were (1) to compare mental health outcomes between ICU and non-ICU HCW; and (2), to determine the factors associated with poor mental health and well-being. These results will help develop appropriate measures to address mental health issues of frontline $\mathrm{HCW}$ in case of future waves or similar outbreaks.

\section{Methods}

\section{Participants and procedures}

A cross-sectional survey was conducted in the HUG in Switzerland to assess levels of anxiety, depression, peritraumatic distress and well-being among HCW. A pretested questionnaire was sent via mailing list servers to all 13,570 HUG employees including physicians, nurses, care assistants, physiotherapists, administrative workers, maintenance workers and patient transporters. ICU $\mathrm{HCW}$ were defined as all the $\mathrm{HCW}$ who were working in the ICU during the first COVID-19 wave. This included regular ICU HCW but also $\mathrm{HCW}$ from other units who had to join the ICU to strengthen the teams during the pandemic. Each employee received an invitation via email to participate in the study. The email contained information on the purpose of the study and a link to the online questionnaire via the RedcapTM ${ }^{\circledR}$ platform. Participation in the study was on a voluntary basis and completely anonymous. Data were collected from May 28th, 2020, to July 7th, 2020, right at the end of the first wave of COVID-19 disease, which ended mid-May in Switzerland.

The questionnaire included questions on socio-demographic data including age, sex, nationality, country of domicile, marital status, number of children, occupation, percentage of work and type of schedule (regular/irregular). It was also assessed whether the person or one of his relatives had had the coronavirus and whether they were afraid of catching it at work or passing it on to their loved ones. The questionnaire included questions on lifestyle changes as assessed by an analog scale, including changes in alcohol consumption, smoking habits, eating patterns, levels of exercise and sleeping habits. The psychiatric scales used were the Generalized Anxiety Disorder 7 items (GAD-7), the Patient Health Questionnaire 9 items (PHQ-9) and the Peritraumatic Distress Inventory (PDI). In order to assess well-being, the World Health Organization Well-Being Index (WHO-5) was used [13-16].

The GAD-7 is a measurement tool that has been validated for screening of generalized anxiety disorder [13]. It uses 7 questions to establish the severity of anxiety and results are interpreted as follows: 0 to 4: no anxiety, 5 to 9: mild anxiety, 10 to 14 : moderate anxiety, 15 to 21 : 
severe anxiety [13]. In order to define the presence of symptoms of anxiety, we used a cut-off score $\geq 5$.

The PHQ-9 is a validated score that evaluates depression through questions regarding the last 2 weeks [16]. The interpretation of the score is the following 0 to 4 : no depression, 5 to 9: mild depression, 10 to 14: moderate depression, 15 to 19 : moderately severe depression, 20 to 27: severe depression [16]. The presence of symptoms of depression was defined with a PHQ-9 score $\geq 5$.

The PDI is a validated score that was designed to evaluate peritraumatic distress through 13 questions regarding the participant's emotional and physiological distress experienced during and immediately after a traumatic event $[17,18]$. PDI $\geq 14$ has been shown to predict PTSD one month after a traumatic event $[14,17]$, which has been defined here as the COVID-19 pandemic in Geneva.

The WHO-5 index is a screening tool based on a 5 -question assessment that has been validated to assess participant well-being and has been shown to be negatively correlated with psychometric tool scores used in psychiatry to assess depression and suicidality $[15,19]$. The score goes from 0 to 100 with 0 being the worst and 100 the best possible well-being score. A score below 50 speaks for low well-being [19].

This study has been approved by Geneva's Regional Research Ethics Committee (BASEC ID 2020-00935).

\section{Statistical analyses}

The analytical sample included ICU and non-ICU HCW who agreed to complete the questionnaire. We performed descriptive analyses of socio-demographic variables according to ICU status. For our first objective, we compared mental health (anxiety/depression symptoms and peritraumatic distress), well-being (treated as continuous scores) and changes in lifestyles (exercise, diet, sleeping habits and substance use; as categorical variables) in ICU HCW, using simple linear regression models and Chisquare tests, accordingly. Per our second objective, we compared the mental health and well-being scores and changes in lifestyle between ICU and non-ICU HCW, by performing a series of $t$-tests and Chi-square tests. Moreover, we performed a series of multivariate linear regression models with ICU status as our main independent variable. All analyses were performed using RStudio (version 1.3.1073). Two-tailed $p$-values at 0.05 were considered statistically significant. Due to the low number of missing data, a complete case analysis was applied.

\section{Results}

\section{Demographic characteristics of the sample population}

Of the 13,570 employees invited to take part in the study, $3461(25 \%)$ accepted to participate. The response rate was much higher among ICU HCW with 352 answers to the survey, corresponding to a response rate of $69 \%$ $(352 / 510)$, compared to $24 \%$ of the response rate among non-ICU HCW $(3109 / 13,060)$ (Table 1, Additional file 1: Figure S1).

Seventy-four percent of participants were women (2561/3461) and age ranged from 18 to 65 years. Sevenhundred and sixty-seven (22.2\%) participants were single and 2215 (64\%) were married. Regarding occupational categories 1341 (38.8\%) participants were nurses, 438 (12.7\%) were physicians, 261 (7.5\%) were care assistants and $1420(41 \%)$ had other functions such as administrative workers. Among the study population, 1949 (56.4\%) reported having had changes in their schedule during the pandemic and 875 (25.3\%) reported being overworked. Regarding coronavirus issues, 833 (24.1\%) participants reported having a relative who had been infected with SARS-CoV-2, 779 (22.5\%) feared getting infected with SARS-CoV-2 and 1592 (46.1\%) were afraid of transmitting it. Further details are illustrated in Table 1.

\section{Prevalence of anxiety, depression, peritraumatic distress and low well-being for ICU HCW}

352 ICU HCW including 198 (56\%) nurses, 68 (19\%) physicians, $54(15 \%)$ other workers and 32 (9\%) care assistants responded to the questionnaire on psychiatric assessment (Table 2). The average GAD-7 score was 6.1 (SD 4.8), indicating mild anxiety, with 25 (7.1\%) of the GAD-7 score reflecting severe anxiety and 49 (13.9\%) moderate anxiety. The average PHQ-9 score was 6.4 (SD 5) reflecting mild depression, with $6(1.7 \%)$ of the population having a score predicting severe depression, 24 (6.8\%) moderate-to-severe depression and 54 (15.3\%) moderate depression. No statistical differences were found in the symptoms of anxiety and depression by occupational category. Regarding PDI, the mean score was 8.8 (SD 7.4) and 76 (21.6\%) of the ICU HCW had a score $\geq 14$ that put them at risk of developing PTSD at one month. The PDI was statistically different by occupational category with a mean score of 8.4 (SD 7.7) for physicians, 9 (SD 7.3) for nurses, 11.7 (SD 8.9) for care assistants, 6.7 (SD 5.6) for other $\mathrm{HCW}$. The average WHO-5 score was 53.3 (SD 23.8) with 145 (41.2\%) of the population having a WHO-5 below 50 reflecting low well-being. The WHO-5 score was statistically different by occupational category with a mean score of 56.8 (SD 21.8) for physicians, 50.2 (SD 25.2) for nurses, 55 (SD 23) for care assistants, 59.3 (SD 19.6) for other HCW.

\section{Lifestyle changes descriptions for ICU HCW}

Regarding lifestyle factors among ICU HCW (Table 3), 159 respondents (45.2\%) reported sleeping less than usual, 114 (32.4\%) eating more. Concerning physical exercise, 164 (46.6\%) reported doing less sport than 
Table 1 Descriptive characteristics of participants $(n=3461)$

\begin{tabular}{|c|c|c|c|}
\hline & Total & ICU & Non-ICU \\
\hline Overall & $3461(100)$ & $352(10)$ & $3109(90)$ \\
\hline \multicolumn{4}{|l|}{ Sex } \\
\hline Women, $n$ (\%) & $2561(74)$ & $234(66.5)$ & $2327(74.9)$ \\
\hline Men, $n(\%)$ & $897(26)$ & $118(33.5)$ & $779(25.1)$ \\
\hline \multicolumn{4}{|l|}{ Age } \\
\hline $18-29$ years, $n(\%)$ & $402(11.6)$ & $39(11)$ & $363(11.7)$ \\
\hline 30-39 years, $n(\%)$ & $815(23.6)$ & $130(36.9)$ & $685(22)$ \\
\hline 40-49 years, $n(\%)$ & $1032(29.8)$ & $97(27.6)$ & $935(30.1)$ \\
\hline $50-59$ years, $n(\%)$ & $1049(30.3)$ & $75(21.3)$ & $974(31.3)$ \\
\hline$\geq 60$ years, $n(\%)$ & $163(4.7)$ & $11(3.1)$ & $152(4.9)$ \\
\hline \multicolumn{4}{|l|}{ Marital status } \\
\hline Single, $n(\%)$ & $767(22.2)$ & $85(24.2)$ & $682(21.9)$ \\
\hline Married, $n(\%)$ & $2215(64)$ & $242(68.7)$ & $1973(63.5)$ \\
\hline Divorced, $n(\%)$ & $451(13)$ & $25(7.1)$ & $426(13.7)$ \\
\hline Widow(-er), n (\%) & $27(0.8)$ & $0(0)$ & $27(0.9)$ \\
\hline \multicolumn{4}{|l|}{ Minor dependent children } \\
\hline Yes, $n(\%)$ & $1635(47.2)$ & $185(52.6)$ & $1450(46.6)$ \\
\hline No, $n(\%)$ & $1826(52.8)$ & $167(47.4)$ & $1659(53.4)$ \\
\hline \multicolumn{4}{|l|}{ Profession } \\
\hline Physician, $n$ (\%) & $438(12.7)$ & $68(19.3)$ & $370(11.9)$ \\
\hline Nurse, $n(\%)$ & $1341(38.8)$ & $198(56.3)$ & $1143(36.8)$ \\
\hline Care assistant, $n(\%)$ & $261(7.5)$ & $32(9.1)$ & $229(7.4)$ \\
\hline Others, $n(\%)$ & $1420(41)$ & $54(15.3)$ & $1366(43.9)$ \\
\hline \multicolumn{4}{|l|}{ Employment rate } \\
\hline Before the pandemic, \%(SD) & $86(17)$ & $87.5(16)$ & $85.8(17.1)$ \\
\hline During the pandemic, \%(SD) & $87(16.2)$ & $90.1(15)$ & $86.6(16.3)$ \\
\hline \multicolumn{4}{|c|}{ Schedule change during the pandemic } \\
\hline Yes, $n(\%)$ & $1949(56.4)$ & $297(84.4)$ & $1652(53.2)$ \\
\hline No, $n(\%)$ & $1507(43.6)$ & $55(15.6)$ & $1452(46.7)$ \\
\hline \multicolumn{4}{|c|}{ Change in workload during the pandemic } \\
\hline Less workload than usual, $n$ (\%) & $512(14.8)$ & $60(17.1)$ & $452(14.6)$ \\
\hline Overload, $n(\%)$ & $875(25.3)$ & $104(29.6)$ & $771(24.8)$ \\
\hline Same workload as usual, $n$ (\%) & $2067(59.8)$ & $187(53.3)$ & $1880(60.6)$ \\
\hline \multicolumn{4}{|l|}{ Country of residence } \\
\hline Switzerland, $n(\%)$ & $1927(56)$ & $167(47.6)$ & $1760(56.9)$ \\
\hline France, $n(\%)$ & $1516(44)$ & $184(52.4)$ & $1332(43.1)$ \\
\hline \multicolumn{4}{|c|}{ Relatives who have had COVID-19 disease } \\
\hline Yes, $n(\%)$ & $833(24.1)$ & $68(19.3)$ & $765(24.6)$ \\
\hline No, $n(\%)$ & $2628(75.9)$ & $284(80.7)$ & $2344(75.4)$ \\
\hline \multicolumn{4}{|l|}{ Fear of catching COVID-19 disease } \\
\hline Yes, $n(\%)$ & $779(22.5)$ & $75(21.3)$ & 704 (22.6) \\
\hline Rather yes, $n(\%)$ & $892(25.8)$ & $90(25.6)$ & $802(25.8)$ \\
\hline Rather no, $n(\%)$ & $1164(33.6)$ & $122(34.7)$ & $1042(34.5)$ \\
\hline No, $n(\%)$ & $626(18.1)$ & $65(18.4)$ & $561(18.1)$ \\
\hline \multicolumn{4}{|c|}{ Fear of transmitting COVID-19 disease } \\
\hline Yes, $n(\%)$ & $1592(46.1)$ & $195(55.4)$ & $1397(45)$ \\
\hline Rather yes, $n(\%)$ & $1017(29.4)$ & $94(26.7)$ & $923(29.7)$ \\
\hline Rather no, $n(\%)$ & $553(16)$ & $42(11.9)$ & $511(16.5)$ \\
\hline No, $n(\%)$ & $293(8.5)$ & $21(6)$ & $272(8.8)$ \\
\hline
\end{tabular}

Table 1 (continued)

\begin{tabular}{|c|c|c|c|}
\hline & Total & ICU & Non-ICU \\
\hline \multicolumn{4}{|c|}{ Fear of working with COVID-19 patients } \\
\hline Yes, $n(\%)$ & $682(19.7)$ & $112(31.8)$ & $570(18.3)$ \\
\hline Rather yes, $n(\%)$ & $1097(31.7)$ & $120(34.1)$ & $977(31.4)$ \\
\hline Rather no, $n(\%)$ & $846(24.4)$ & $99(28.1)$ & $747(24.1)$ \\
\hline No, $n(\%)$ & $836(24.2)$ & $21(6)$ & $815(26.2)$ \\
\hline \multicolumn{4}{|c|}{ Use of any psychological support } \\
\hline Yes, $n(\%)$ & $420(12.1)$ & $98(27.8)$ & $322(10.4)$ \\
\hline No, $n(\%)$ & $3041(77.9)$ & $254(72.2)$ & $2787(89.6)$ \\
\hline \multicolumn{4}{|c|}{ Hotel accommodation during the pandemic } \\
\hline Yes, $n(\%)$ & $231(6.7)$ & $84(24.9)$ & $147(4.7)$ \\
\hline No, $n(\%)$ & $3230(93.3)$ & $268(76.1)$ & $2962(95.3)$ \\
\hline
\end{tabular}

Values were expressed in numbers and percentages

usual. Considering alcohol and tobacco consumption, 78 (22.2\%) respondents reported an increase in alcohol consumption and $53(15 \%)$ reported an increase in tobacco consumption. No statistically significant differences were shown in the different occupational categories for lifestyle changes.

\section{Comparison of mental health outcomes, well-being} and lifestyle changes between ICU HCW and non-ICU HCW Figure 1 compares ICU and non-ICU HCW regarding mental health, well-being and lifestyle changes. The mean GAD-7 and PHQ-9 scores were higher in ICU compared to non-ICU HCW, while ICU HCW reported lower wellbeing scores (WHO-5). No significant difference in PDI score was observed. In terms of lifestyle habits, working in the ICU rather than in another hospital unit was associated with having a change in diet $(p<0.01)$, in sleeping patterns $(<0.01)$ and in alcohol consumption $(p<0.01)$. Details can be found in Additional file 1: Table S1.

\section{Identification of risk factors of poor mental health outcome} and low well-being among ICU and non-ICU HCW

Table 4 presents the factors that have been found to be associated with an increase in GAD-7, PHQ-9 and PDI score and a decrease in the WHO-5 scale among all the employees (ICU HCW and non-ICU HCW). We were able to identify common factors associated with the 4 scores that we can classify into several categories: (1) socio-demographic factors: being female; (2) working environmental factors: being overloaded with work; (3) anxiety towards COVID-19: fear of catching and transmitting it, anxiety of working in contact with COVID-19 patients; (4) somatic symptoms: eating less, sleeping disorders; (5) impact on consumptions: drinking more alcohol. Details of multivariable models are available in the Additional file 1: Table S2. 
Table 2 Score descriptions for ICU HCW $(n=352)$

\begin{tabular}{|c|c|c|c|c|c|c|}
\hline & \multicolumn{5}{|c|}{$\mathrm{ICU}(n=352)$} & \multirow[t]{2}{*}{$p$ value } \\
\hline & Total & Physicians & Nurses & Care assistants & Others & \\
\hline Overall, $n(\%)$ & $352(100)$ & $68(19)$ & $198(56)$ & $32(9)$ & $54(15)$ & \\
\hline \multicolumn{7}{|l|}{ GAD-7 } \\
\hline Mean score (SD) & $6.1(4.8)$ & $6.0(5.0)$ & $6.3(5.0)$ & $6.5(4.7)$ & $5.0(4.1)$ & 0.33 \\
\hline Minimal anxiety, $n(\%)$ & $190(54)$ & $41(60.3)$ & $98(49.5)$ & $14(43.7)$ & $37(68.5)$ & 0.32 \\
\hline Mild anxiety, $n$ (\%) & $88(25)$ & $12(17.7)$ & $55(27.8)$ & $11(34.4)$ & $10(18.5)$ & \\
\hline Moderate anxiety, $n(\%)$ & 49 (13.9) & $10(14.7)$ & $30(15.1)$ & $4(12.5)$ & $5(9.3)$ & \\
\hline Severe anxiety, $n(\%)$ & $25(7.1)$ & $5(7.3)$ & $15(7.6)$ & $3(9.4)$ & $2(3.7)$ & \\
\hline \multicolumn{7}{|l|}{ PHQ-9 } \\
\hline Mean score (SD) & $6.4(5.0)$ & $6.4(5.5)$ & $6.7(5.2)$ & $6.8(4.9)$ & $5.1(3.8)$ & 0.20 \\
\hline Minimal depression, $n(\%)$ & $189(53.7)$ & $40(58.8)$ & $97(49)$ & $16(50)$ & $36(66.7)$ & 0.34 \\
\hline Mild depression, $n(\%)$ & $79(22.4)$ & $12(17.7)$ & $47(23.7)$ & $8(25)$ & $12(22.2)$ & \\
\hline Moderate depression, $n(\%)$ & $54(15.3)$ & $10(14.7)$ & $35(17.7)$ & $5(15.6)$ & $4(7.4)$ & \\
\hline Moderately severe depression, $n(\%)$ & $24(6.8)$ & $3(4.4)$ & $16(8.1)$ & $3(9.4)$ & $2(3.7)$ & \\
\hline Severe depression, $n$ (\%) & $6(1.7)$ & $3(4.4)$ & $3(1.5)$ & $0(0)$ & $0(0)$ & \\
\hline \multicolumn{7}{|l|}{ PDI } \\
\hline Mean score (SD) & $8.8(7.4)$ & $8.4(7.7)$ & $9.0(7.3)$ & $11.7(8.9)$ & $6.7(5.6)$ & 0.02 \\
\hline Not a risk for PTSD, $n(\%)$ & $276(78.4)$ & $56(82.4)$ & $151(76.3)$ & $21(66)$ & $48(88.9)$ & 0.05 \\
\hline At risk for PTSD, $n(\%)$ & $76(21.6)$ & $12(17.6)$ & $47(23.7)$ & $11(34)$ & $6(11.1)$ & \\
\hline \multicolumn{7}{|l|}{ WHO-5 } \\
\hline Mean score (SD) & $53.3(23.8)$ & $56.8(21.8)$ & $50.2(25.2)$ & $55.0(23.0)$ & $59.3(19.6)$ & 0.03 \\
\hline$<50, n(\%)$ & $145(41.2)$ & $22(32)$ & $95(48)$ & $14(44)$ & $14(26)$ & 0.01 \\
\hline$\geq 50 n(\%)$ & $207(58.8)$ & $46(68)$ & $103(52)$ & $18(56)$ & $40(74)$ & \\
\hline
\end{tabular}

Values were expressed in numbers and percentages; mean values and standard deviation

WHO-5 World Health Organization Well-Being Index, GAD-7 7-item Generalized Anxiety Disorders, PHQ-9 9 items Patient Health Questionnaire, PDI Peritraumatic Distress Inventory, NA not available

\section{Discussion}

This cross-sectional study included a total of 3,461 HCW of which 352 were ICU HCW during the first wave of the COVID-19 crisis. Among ICU HCW, $41 \%$ had low well-being, $46 \%$ had anxiety symptoms, $46 \%$ had symptoms of depression and $22 \%$ had peritraumatic distress. Scores for risk of depression, anxiety and low well-being were statistically more pathological in the ICU than in other hospital units. A change in lifestyle factors was also highlighted with an increase in alcohol consumption and a modification of eating and sleeping habits among ICU $\mathrm{HCW}$. In the entire studied population (ICU and nonICU HCW), several factors were found to be associated with symptoms of anxiety, depression, peritraumatic distress and low well-being: being female, the fear of catching and transmitting COVID-19, anxiety of working in contact with COVID-19 patients, being overloaded with work, eating less, increased alcohol consumption and sleeping disorders.

With regard to anxiety and depression among ICU HCW, the present study confirms the findings of several studies conducted during the pandemic reporting a prevalence of anxiety ranging from 48 to $50.4 \%$ and depression ranging from 16 to $30.4 \%$ among ICU HCW $[6,8]$. Comparison with these studies' results should be made with caution as different scores were used. Many factors may explain the high prevalence of depression and anxiety symptoms described in ICU HCW during this crisis. Firstly, the high rate of anxiety could be explained by the fact that COVID-19 appeared unpredictable and potentially lethal. HCW were exposed to this uncertainty which not only concerned their patients, but themselves as well, making them feel powerless. Secondly, the media coverage of the events with the announcement in February 2020 of more than 3000 caregivers infected with COVID-19 in China could have been a source of stress for HCW [20]. Thirdly, during this first wave of COVID-19, the HUG mainly treated COVID-19 patients while patients with other pathologies were hospitalized in the surrounding hospitals. This distribution could have been a source of anxiety and depression for HCW who were continuously exposed to this extreme situation. No differences in the symptoms of anxiety and depression by 
Table 3 Lifestyle changes descriptions for ICU HCW $(n=352)$

\begin{tabular}{|c|c|c|c|c|c|c|}
\hline & \multicolumn{5}{|c|}{$\operatorname{ICU}(n=352)$} & \multirow[t]{2}{*}{$p$ value } \\
\hline & Total & Physicians & Nurses & Care assistants & Others & \\
\hline Overall, $n(\%)$ & $352(100)$ & $68(19)$ & $198(56)$ & $32(9)$ & $54(15)$ & \\
\hline Sleeping habits & & & & & & 0.26 \\
\hline Less than usual, $n(\%)$ & $159(45.2)$ & $24(35.3)$ & $95(48)$ & $13(40.6)$ & $27(50)$ & \\
\hline Same as usual, $n$ (\%) & $95(27)$ & $26(38.2)$ & $48(24.2)$ & $7(21.9)$ & $14(25.9)$ & \\
\hline More than usual, $n(\%)$ & $98(27.8)$ & $18(26.5)$ & $55(27.8)$ & $12(37.5)$ & $13(24.1)$ & \\
\hline Eating habits & & & & & & 0.21 \\
\hline Less than usual, $n(\%)$ & $47(13.4)$ & $5(7.4)$ & $28(14.1)$ & $7(21.9)$ & $7(12.9)$ & \\
\hline Same as usual, $n(\%)$ & $191(54.2)$ & $41(60.3)$ & $100(50.6)$ & $20(62.5)$ & $30(55.6)$ & \\
\hline More than usual, $n(\%)$ & $114(32.4)$ & $22(32.3)$ & $70(35.3)$ & $5(15.6)$ & $17(31.5)$ & \\
\hline Exercise & & & & & & 0.07 \\
\hline Less than usual, $n(\%)$ & $164(46.6)$ & $30(44.1)$ & $103(52)$ & $12(37.5)$ & $19(35.2)$ & \\
\hline Same as usual, $n(\%)$ & $131(37.2)$ & $22(32.4)$ & $71(35.9)$ & $12(37.5)$ & $26(48.1)$ & \\
\hline More than usual, $n(\%)$ & $57(16.2)$ & $16(23.5)$ & $24(12.1)$ & $8(25)$ & $9(16.7)$ & \\
\hline Alcohol & & & & & & 0.06 \\
\hline Less than usual, $n(\%)$ & $29(8.2)$ & $3(4.4)$ & $16(8.1)$ & $5(15.6)$ & $5(9.3)$ & \\
\hline Same as usual, $n$ (\%) & $245(69.6)$ & $44(64.7)$ & $135(68.2)$ & $25(78.1)$ & $41(75.9)$ & \\
\hline More than usual, $n(\%)$ & $78(22.2)$ & $21(30.9)$ & $47(23.7)$ & $2(6.3)$ & $8(14.8)$ & \\
\hline Tobacco & & & & & & 0.16 \\
\hline Less than usual, $n(\%)$ & $14(4)$ & $3(4.4)$ & $7(3.5)$ & $3(9.4)$ & $1(1.8)$ & \\
\hline Same as usual, $n$ (\%) & $285(81)$ & 55 (80.9) & $158(79.8)$ & $22(68.7)$ & $50(92.6)$ & \\
\hline More than usual, $n(\%)$ & $53(15)$ & $10(14.7)$ & $33(16.7)$ & $7(21.9)$ & $3(5.6)$ & \\
\hline
\end{tabular}

Values were expressed in numbers and percentages

occupational category were highlighted in our study. This might be explained by the fact that in our hospital, the staff that had been widely recruited has made it possible to maintain good working conditions with an average of one nurse for every two patients in the ICU. Studies $[6,11]$ describing a more important role of anxiety and depression in nurses did not mention this type of information thus making this interpretation harder to ascertain.

We assessed peritraumatic distress in ICU HCW because we suspected that the COVID-19 pandemic would potentially expose them to traumatic events [21], such as numerous and unpredictable deaths [22]. Our study showed that $22 \%$ of ICU HCW displayed peritraumatic distress. Peritraumatic distress, which is defined as the emotional and physiological distress experienced during and immediately after a traumatic event, is a known risk factor of developing PTSD one month after a traumatic event [14]. Indeed, a French study observed that $27 \%$ of ICU HCW experienced PTSD in the context of the pandemic [6]. These results highlight the important risk of PTSD for ICU HCW and the need to implement preventative measures to support them. Protective factors for PTSD include good coping strategies in stressful situations, primary prevention, training before a traumatic event and positive social support following a traumatic event $[23,24]$.

Another objective of our study was to assess whether ICU HCW had suffered more from the pandemic compared to non-ICU HCW. Indeed, we showed that ICU HCW had more symptoms of anxiety, depression and lower well-being. The ICU is known to be a difficult work environment due to heavy workloads, exposure to critically ill patients, daily confrontation with death and irregular working hours [10]. Even outside major crises, ICU HCW have been shown to be more prone to anxiety and depression compared to staff from other units [10, 25]. During catastrophic situations, ICU HCW tend to leave their needs aside to meet the needs of patients [6]. In an already stressful work environment, poorer mental health outcomes can be expected to be exacerbated by the stress caused by the pandemic with many unknowns, the fear of catching and transmitting the virus, the high influx of patients, the fear of not having enough resources and changes in work habits. However, unlike what might have been expected, no significant difference was found in peritraumatic distress between ICU HCW and nonICU HCW. As the exposition to traumatic events of unexpected and numerous deaths occurred in all hospital units and not only in the ICU, this could explain the 
A.
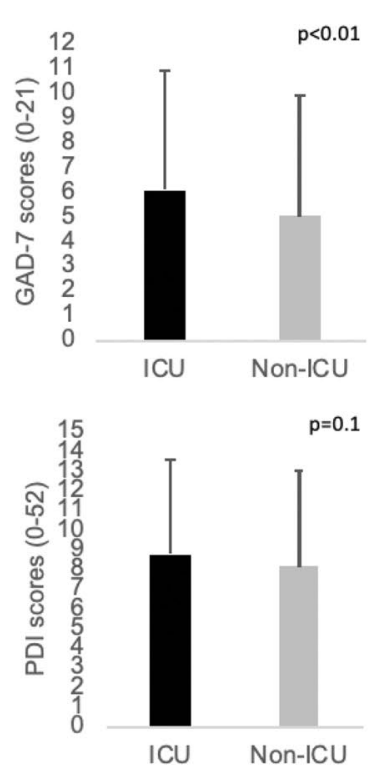

B.
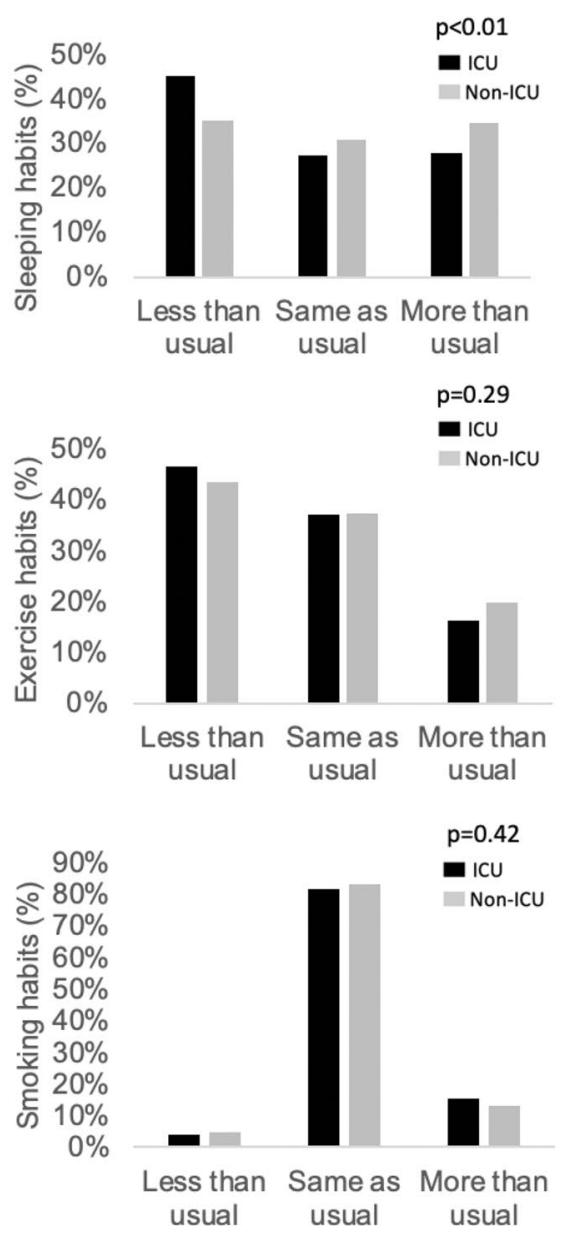
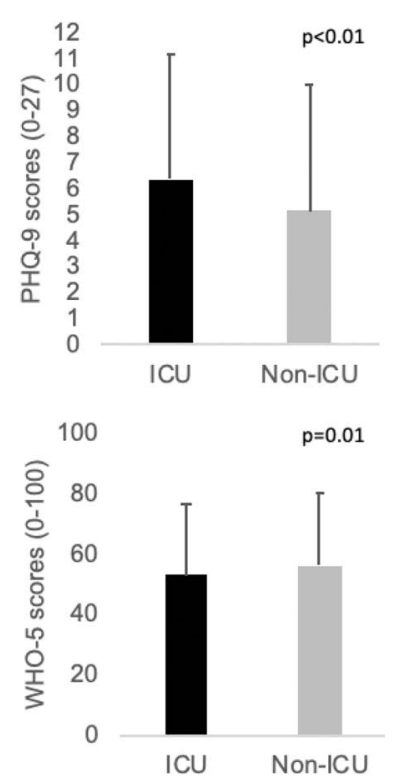

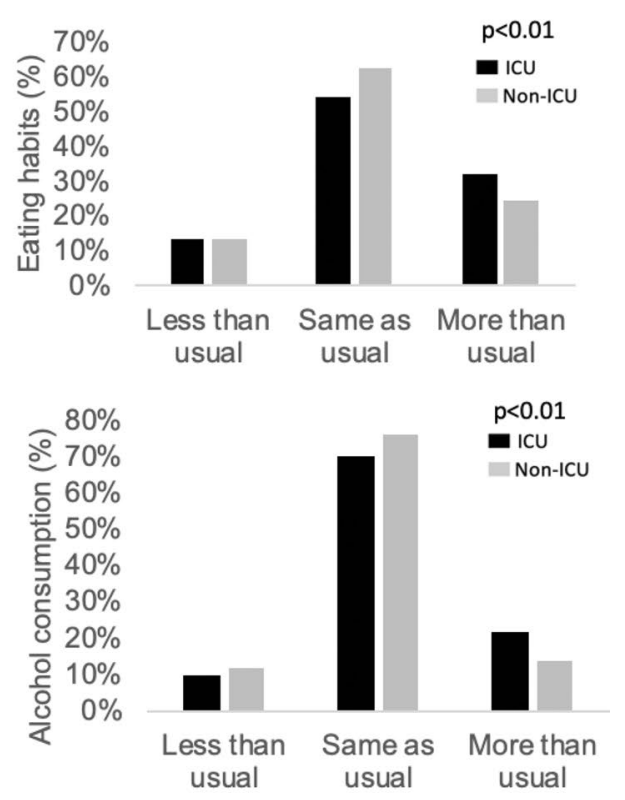

Fig. 1 Comparison of psychiatric scores, well-being and lifestyle changes between ICU and non-ICU HCW. A Comparison of psychiatric scores and well-being between ICU and non-ICU HCW. B Comparison of lifestyle changes between ICU and non-ICU HCW 
Table 4 Identification of risk factors of high psychiatric scores and low well-being scale among ICU and non-ICU HCW

\begin{tabular}{|c|c|c|c|}
\hline GAD-7 & PHQ-9 & PDI & WHO-5 \\
\hline Female & Older & Older & Female \\
\hline No minor children & Female & Female & Single \\
\hline Work overload & Single & Single & Work overload \\
\hline Fear of catching COVID-19 & Work overload & Work overload & Occupation \\
\hline Fear of transmitting COVID-19 & Fear of catching COVID-19 & Care assistant & Relatives affected by COVID-19 \\
\hline \multirow{8}{*}{$\begin{array}{l}\text { Stress of working in Contact wi } \\
\text { COVID-19 } \\
\text { Trouble sleeping } \\
\text { Eating less } \\
\text { More alcohol }\end{array}$} & Fear of transmitting COVID-19 & Relatives affected by COVID-19 & Fear of catching COVID-19 \\
\hline & \multirow{7}{*}{$\begin{array}{l}\text { Stress of working in contact with } \\
\text { COVID-19 } \\
\text { Trouble sleeping } \\
\text { Eating less } \\
\text { More alcohol } \\
\text { Less physical exercise }\end{array}$} & Fear of catching COVID-19 & Fear of transmitting COVID-19 \\
\hline & & Fear of transmitting COVID-19 & Stress of working in contact with \\
\hline & & Stress of working in contact with & COVID -19 \\
\hline & & COVID-19 & Trouble sleeping \\
\hline & & Trouble sleeping & Eating less \\
\hline & & Eating less & More alcohol \\
\hline & & More alcohol & Less physical exercise \\
\hline
\end{tabular}

WHO-5 World Health Organization Well-Being Index, GAD-7 7-item Generalized Anxiety Disorders, PHQ-9 9 items Patient Health Questionnaire, PDI Peritraumatic Distress Inventory

lack of difference in peritraumatic distress between the two groups. A feature of our study was to include a significant rate of "other workers" ( $41 \%$ of our total sample), such as administrative workers, while other studies disregarded this population. Interestingly, a sensitivity analysis showed that this group of workers [over-represented in non-ICU (44\%) vs. ICU (15\%)] did not influence the differences observed in mental health and well-being outcomes between ICU and non-ICU HCW.

The present study showed important changes in lifestyle behavior in $\mathrm{HCW}$ and ICU HCW appeared to have increased their alcohol consumption more than nonICU HCW. Studies have shown that exposure to traumatic events, such as terrorist attacks, natural events or in this case the COVID-19 outbreak, is associated with increased alcohol consumption [26]. This has also been shown in the general population where an increase in stress-related alcohol consumption during the COVID19 pandemic has been highlighted [27]. To the best of our knowledge, we are the first to raise this point in HCW in the context of the pandemic and to show its association with more anxiety, depression, peritraumatic distress and low well-being in this setting. The potential for alcohol abuse by exposed HCW must be recognize as it may have implications for their physical health and should be followed up.

Another objective was to identify risk factors for worse mental health outcomes in all the HCW (ICU and nonICU). Our study significantly showed that ICU HCW suffered more psychologically than non-ICU HCW. Interestingly, after adjustment for socio-demographic variables and lifestyle behaviors, working in the ICU was no longer an independent predictor of poor mental health outcomes. However, we were able to identify several independent risk factors for poor mental health outcomes in all the HCW (ICU and non-ICU). These factors were classified into 5 categories. (1) socio-demographic factors: female have been found to have more anxiety, depression and peritraumatic distress in our study, which is an already known risk factor even outside the coronavirus outbreak $[5,7,11]$. (2) Working environmental factors: as previously described in a French study, being overworked was associated with poorer mental health outcome [28]. A strategy that allows for break times and balanced work schedule could help control this factor. (3) Somatic symptoms: sleep disorders and changes in eating habits among HCW were associated with poor mental health outcomes in the context of the COVID-19 crisis. Indeed, several studies have identified sleeping disorders in frontline HCW during the pandemic [11, 29]. (4) Consumptions: our results show that increased alcohol consumption has been found to be independently associated with poor mental health outcome. (5) Fear towards COVID-19: we were able to identify specific risk factors in the context of this pandemic and found that being afraid of catching, transmitting or working with COVID19 patients was associated with higher anxiety, depression and peritraumatic distress. As raised in the Azoulay et al. study, fear leads to general discomfort, fatigue and difficulty in decision-making [8]. Since the feeling of fear among $\mathrm{HCW}$ was frequently reported in several studies, providing regular, accurate and detailed information on the virus, its mode of transmission and associated protective measures, seems crucial $[6,8,20]$. This study reinforces the knowledge on factors associated with poor mental health outcomes during the COVID-19 crisis. In light of these considerations, hospital managers should be able to pay particular attention to $\mathrm{HCW}$ at risk $[8,11$, $12,29]$.

Some limitations in our study need to be acknowledged. The cross-sectional design of our study does not allow us to infer causality between the factors studied 
and the psychic symptoms, but only to find an association. Only $25 \%$ of the total $\mathrm{HCW}$ responded to the study questionnaire and a selection bias is therefore possible; however, the response rate was high among ICU HCW (69\%). Another limitation was our inability to assess psychiatric history or previous trauma. The study was monocentric; however, the HUG is a large hospital consortium comprising eight different sites. Finally, we did not further assess ICU HCW to differentiate between back-up ICU HCW and usual ICU HCW.

\section{Conclusion}

Our results strengthen the findings of previous studies conducted on the mental health of HCW during the COVID-19 outbreak and highlight the high prevalence of anxiety, depression, peritraumatic distress and low wellbeing, especially among ICU HCW. Lifestyle changes in areas such as amount of physical activity, sleeping and eating patterns as well as alcohol and tobacco consumption were also highlighted during the pandemic. Longterm psychological follow-up should be considered for HCW.

\section{Supplementary Information}

The online version contains supplementary material available at https://doi. org/10.1186/s13613-021-00900-x.

Additional file 1: Table S1. Comparison of psychiatric scores, well-being and lifestyle changes between ICU and non-ICU HCW. Table S2. Factors associated with mental health and well-being outcomes. Figure S1.

Flowchart of survey population sampling.

\section{Acknowledgements}

Not applicable.

\section{Authors' contributions}

All authors contributed to the study conception and design. Material preparation, data collection and analysis were performed by GM, AN, JP, SC and HW. The first draft of the manuscript was written by HW and all authors commented on previous versions of the manuscript. All authors read and approve the final manuscript.

\section{Funding}

Niccolò Buetti is currently receiving a mobility grant from Swiss National Science Foundation (grant number: P4P4PM_194449).

\section{Availability of data and materials}

The datasets used and analyzed during the current study are available from the corresponding author on reasonable request.

\section{Declarations}

Ethics approval and consent to participate

Our study was approved by the Swiss ethic committee (BASEC ID 2020-00935) and was conducted in accordance with the Declaration of Helsinki.

\section{Consent for publication}

Participation in the study was voluntary and the questionnaire was completed anonymously. Participants were informed before answering the survey that it would lead to a scientific publication and that they accepted this if they decided to answer the questionnaire.

\section{Competing interests}

The authors declare that they have no competing interests.

\section{Author details}

${ }^{1}$ Intensive Care Unit, Geneva University Hospitals, Geneva, Switzerland. ${ }^{2}$ Psychiatric Liaison and Crisis Intervention Service, Geneva University Hospitals, Geneva, Switzerland. ${ }^{3}$ School of Public Health, University of Montréal, Montreal, QC, Canada. ${ }^{4}$ Infection Control Program and World Health Organization Collaborating Centre on Patient Safety, University Hospitals and Faculty of Medicine, University of Geneva, Geneva, Switzerland. ${ }^{5}$ Personnel Health Service, Geneva University Hospitals, Geneva, Switzerland. ${ }^{6}$ Arc School of Health, University of Applied Sciences and Arts Western Switzerland, Neuchâtel, Switzerland. ${ }^{7}$ School of Health Sciences, University of Applied Sciences and Arts Western Switzerland, Valais-Wallis, Sion, Switzerland. ${ }^{8}$ Faculty of Medicine, University of Geneva, Geneva, Switzerland. ${ }^{9}$ Pulmonary Division, Geneva University Hospitals, Geneva, Switzerland.

Received: 23 April 2021 Accepted: 4 July 2021

Published online: 10 July 2021

\section{References}

1. Yang Y, Peng F, Wang R, Guan K, Jiang T, Xu G, Sun J, Chang C. The deadly coronaviruses: the 2003 SARS pandemic and the 2020 novel coronavirus epidemic in China, the Company's Public News and Information. J Autoimmun. 2020;109:102487.

2. Zhou P, Lou YX, Wang XG, et al. A pneumonia outbreak associated with a new coronavirus of probable bat origin. Nature. 2020;579:270-3.

3. Primmaz S, Le Terrier C, Suh N, et al. Preparedness and reorganization of care for coronavirus disease 2019 patients in a Swiss ICU: characteristics and outcomes of 129 patients. Crit Care Explor. 2020;2:e0173.

4. Wu P, Fang Y, Guan Z, et al. The psychological impact of the SARS epidemic on hospital employees in China: exposure, risk perception, and altruistic acceptance of risk. Can J Psychiatry. 2009;54:302-11.

5. Elbay RY, Kurtulmuş A, Arpacıoğlu S, Karadere E. Depression, anxiety, stress levels of physicians and associated factors in Covid-19 pandemics. Psychiatry Res. 2020;290:1-5.

6. Caillet A, Coste C, Sanchez R, Allaouchiche B. Psychological impact of COVID-19 on ICU caregivers. Anaesth Crit Care Pain Med. 2020;39:717-22.

7. Rossi R, Socci V, Pacitti F, Di Lorenzo G, Di Marco A, Siracusano A, Rossi A Mental health outcomes among frontline and second-line health care workers during the coronavirus disease 2019 (COVID-19) pandemic in Italy. JAMA Netw open. 2020;3:e2010185.

8. Azoulay E, Cariou A, Bruneel F, et al. Symptoms of anxiety, depression, and peritraumatic dissociation in critical care clinicians managing patients with COVID-19 a cross-sectional study. Am J Respir Crit Care Med. 2020;202:1388-98.

9. Mealer M, Burnham EL, Goode CJ, Rothbaum B, Moss M. The prevalence and impact of post traumatic stress disorder and burnout syndrome in nurses. Depress Anxiety. 2009;26:1118-26.

10. Vandevala T, Pavey L, Chelidoni O, Chang NF, Creagh-Brown B, Cox A. Psychological rumination and recovery from work in intensive care professionals: associations with stress, burnout, depression and health. J Intensive Care. 2017;5:1-8.

11. Lai J, Ma S, Wang Y, et al. Factors associated with mental health outcomes among health care workers exposed to coronavirus disease 2019. JAMA Netw open. 2020;3:e203976.

12. Greenberg N, Weston D, Hall C, Caulfield T, Williamson V, Fong K. Mental health of staff working in intensive care during Covid-19. Occup Med (Lond). 2021;71:62-7.

13. Spitzer RL, Kroenke K, Williams JW, Löwe B. A brief measure for assessing generalized anxiety disorder: the GAD-7. Arch Intern Med. 2006;166:1092-7.

14. Bunnell BE, Davidson TM, Ruggiero KJ. The Peritraumatic Distress Inventory (PDI): FACTOR structure and predictive validity in traumatically injured patients admitted through a Level I trauma center. J Anxiety Disord. 2018;55:8-1. 
15. Blankers T, Fracica E, Molella RG, Franz ZKW, W, Kashyap R, . Measuring well-being of simulation participants using the World Health Organization-5 well-being index. Med Saf Glob Heal. 2016;5:2-5.

16. Kroenke K, Spitzer RL, Williams JBW. The PHQ-9: validity of a brief depression severity measure. J Gen Intern Med. 2001;16:606-13.

17. Chaix B, Delamon G, Guillemasse A, Brouard B, Bibault J-E. Psychological Distress during the COVID-19 pandemic in France: a national assessment of at-risk populations. Gen Psychiatry. 2020;33:1-14.

18. Brunet A, Weiss DS, Metzler TJ, Best SR, Neylan TC, Rogers C, Fagan J, Marmar CR. The peritraumatic distress inventory: a proposed measure of PTSD criterion A2. Am J Psychiatry. 2001;158:1480-5.

19. Topp CW, Østergaard SD, Søndergaard S, Bech P. The WHO-5 well-being index: a systematic review of the literature. Psychother Psychosom. 2015;84:167-76

20. Sun N, Wei L, Shi S, et al. Qualitative study: experienced of caregivers during Covid19. Am J Infect Control. 2020;48:592-8.

21. Pai A, Suris AM, North CS. Posttraumatic stress disorder in the dsm-5: Controversy, change, and conceptual considerations. Behav Sci (Basel). 2017. https://doi.org/10.3390/bs7010007.

22. Benfante A, Di Tella M, Romeo A, Castelli L. traumatic stress in healthcare workers during COVID-19 pandemic: a review of the immediate impact. Front Psychol. 2020. https://doi.org/10.3389/fpsyg.2020.569935.

23. Lauth Lebens $M$, Lauth WG. Risk and resilience factors of post-traumatic stress disorder: a review of current research. Clin Exp Psychol. 2016. https://doi.org/10.4172/2471-2701.1000120.
24. Brewin CR, Andrews B, Valentine JD. Meta-analysis of risk factors for posttraumatic stress disorder in trauma-exposed adults. J Consult Clin Psychol. 2000;68:748-66.

25. Costa DK, Moss M. The cost of caring: emotion, burnout, and psychological distress in critical care clinicians. Ann Am Thorac Soc. 2018;15:787-90.

26. Keyes KM, Hatzenbuehler ML, Grant BF, Hasin DS. Stress and alcohol epidemiologic evidence. Alcohol Res Curr Rev. 2012;34:391-400.

27. Stanton R, To QG, Khalesi S, Williams SL, Alley SJ, Thwaite TL, Fenning AS, Vandelanotte C. Depression, anxiety and stress during COVID-19: Associations with changes in physical activity, sleep, tobacco and alcohol use in Australian adults. Int J Environ Res Public Health. 2020;17:1-13.

28. Azoulay E, De Waele J, Ferrer R, et al. Symptoms of burnout in intensive care unit specialists facing the COVID-19 outbreak. Ann Intensive Care. 2020. https://doi.org/10.1186/s13613-020-00722-3.

29. Pappa S, Ntella V, Giannakas T, Giannakoulis VG. Since January 2020 Elsevier has created a COVID-19 resource centre with free information in English and Mandarin on the novel coronavirus COVID-19. The COVID-19 resource centre is hosted on Elsevier Connect, the company's public news and information. Brain, Behav Immun. 2020;88:901-7.

\section{Publisher's Note}

Springer Nature remains neutral with regard to jurisdictional claims in published maps and institutional affiliations.

\section{Submit your manuscript to a SpringerOpen ${ }^{\circ}$ journal and benefit from:}

- Convenient online submission

- Rigorous peer review

- Open access: articles freely available online

- High visibility within the field

- Retaining the copyright to your article

Submit your next manuscript at $\boldsymbol{\nabla}$ springeropen.com 